\title{
NEW DEVELOPMENTS IN CCD TECHNOLOGY FOR THE UV-EUV SPECTRAL RANGE
}

\author{
Giovanni Bonanno
}

Catania Astrophysical Observatory

\begin{abstract}
Lately, Charge Coupled Device (CCD) detectors have had great advances both in the visible and in the X-ray spectral range. However, the technology applied to these devices in the ultraviolet (UV) spectral region has not developed as well, because of some problems connected with the interaction between UV radiation and the materials typically used in semiconductor technology. In our laboratory the ultraviolet response of some UV-enhanced CCDs has been investigated. In particular, the quantum efficiency of coronene and lumigen coated and of back-illuminated ion implanted CCDs have been measured in the $304-11000 \AA$ spectral range. Very interesting results have been found, mainly for a one ion implanted CCD with quantum efficiency values of more than $60 \%$ at $304 \AA$. Some measurements of the response uniformity of this spectral region have also been made. The results obtained encourage the possible use of these detectors in ultraviolet astronomy with very good performance.
\end{abstract}

\section{INTRODUCTION}

Charge Coupled Devices (CCDs) are widely used as imaging detectors both in the optical and in the X-ray spectral range. In many observatories various CCD cameras are operated in the optical. There are very good results in laboratories such as JPL (Janesick et al. 1989, 1990) and Lockheed Palo Alto Research (Stern et al. 1986) in the USA, and Space Research at Utrecht (Verhoeve et al. 1991, Bailey et al. 1990) and Leicester University (McCarthy and Wells 1992) in Europe. The use of these devices in the ultraviolet (UV) spectral region is obviously very attractive especially for space instruments. But, CCD technology in this spectral region presents some problems connected with the interaction between UV radiation and silicon. As shown in Fig. 1 the absorption depth in the $1000-4000 \AA$ region is very low and then the interaction occurs on the first layers of the material. For example, the relatively thick gate oxide layer $(500$ - $1200 \AA$ ) on the CCD front face strongly absorbs UV radiation, thus it is impossible to use front-illuminated CCDs for direct detection of UV radiation.

Many solutions have been tried: the most common technique is to sublimate a phosphor under vacuum conditions, such as "lumigen" on the sensitive surface (Bredthauer et al. 1988). This is done both for front-illuminated and thinned back-illuminated CCDs, down-converting the UV radiation and the absorption due to the thick polysilicon gate can be avoided. In backilluminated CCDs, other problems have to be solved. After the device is thinned, a "native" back-oxide layer forms naturally which contains a lot of "dangling bonds" creating surface states. Since there are positive charges associated with them, a depletion region at the back of the CCD forms (backside well) and, as stated before, owing to the extremely short path length ( $<100 \AA)$ in silicon for UV photons, the charge is photogenerated very near to the CCD backside, so that the recombination is highly probable and a lot of signal charge can be lost. 


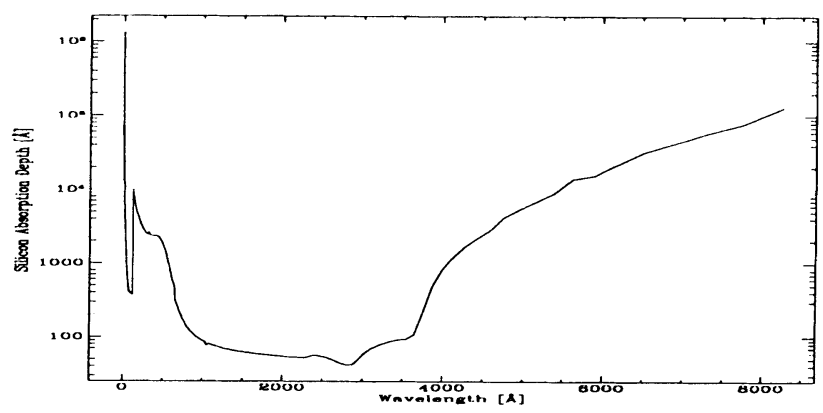

Fig. 1. Silicon Absorption Depth vs wavelength in the $1.2-8500 \AA$ region.

Some methods have been developed to reduce surface state problems using different "backaccumulation" techniques. They are based on the hypothesis that the back surface potential can be pinned by heavily populating it with holes which create an electric field that directs the photogenerated charges away from the back surface (Janesick et al. 1989). The methods can be divided into three categories: backside charging or UV flooding (Janesick et al. 1985), flash gate techniques (Janesick et al. 1989) and ion-implantation and successive laser-annealing.

The last technique is based on the fact that the effect of a $\mathrm{p}^{+}$implantation on the backside lowers the backside well width (Janesick et al. 1985) to values of the order of $10 \AA$ or less. Moreover, the implantation damages the crystal lattice sites, so that it is necessary to anneal and reconstruct the crystal structure. To do this a pulsed laser annealing of the CCD back surface is adopted (Bailey et al. 1990); if the process is optimal, at the end, no significant residual backside well should be present.

There is only one small problem that can be still present: if some ions penetrate deeply into the surface they cannot be reached by annealing; in such a way, the associated crystal damage acts like traps for the charge signal, reducing the $\mathrm{QE}$ performances. Thus, to minimize this problem, a low energy ion-implantation technique must be used.

Another new technique named "anodic etching" has been developed by English Electric Valve (EEV) and it consists in the removal of the oxide layer by selective etching. This technique allows the boron-implanted layer to be thinned in a controlled way (by means of an appropriate voltage during the anodization). Anodic etching could be applied either before or after laser annealing. Thus the deep penetrated boron atoms, which are left unannealed in the standard technique, could be reached now. Another technique that is under investigation at EEV is the ultra low energy boron implantation $(\mathrm{E}<2 \mathrm{KeV})$ that leaves no unannealed boron and can be combined with "anodic etching".

In the following sections, I will describe the results obtained from measurements of the $\mathrm{QE}$ and uniformity response performances in the 300 - $11000 \AA$ spectral range of some UVenhanced EEV CCDs. I selected one per type: one is front-illuminated and phosphor coated, the others are thinned back-illuminated with the surface treated as: phosphor coated, ionimplanted and ion-implanted with the "anodic etching". 


\section{CCD CHIPS}

We have tested several EEV CCDs of the CCD02-06 (385 x 576 pixels, $22 \mu$ m pixel size) series. Table 1 summarizes the relevant characteristics of the four selected CCDs.

TABLE 1

Characteristics of Four Selected CCDs

\begin{tabular}{|c|c|c|c|}
\hline $\begin{array}{l}\text { CCD } \\
\text { TYPE }\end{array}$ & ILL. & SURFACE TREATMENT & NOTES \\
\hline Thick & Front & Lumigen vacuum sublimation & scientific grade \\
\hline Thinned & Back & Coronene coating & scientific grade \\
\hline Thinned & Back & Ion-implanted laser annealed & $\mathrm{E}=10 \mathrm{KeV}, \mathrm{C} .=10^{15} \mathrm{~cm}^{2}$ \\
\hline Thinned & Back & Ion-implanted laser annealed & as above, anodic etched \\
\hline
\end{tabular}

The back-illuminated ion-implanted CCDs are of the same type as those furnished by EEV for the characterization tests of the detector for the Reflection Grating Spectrometer on the XMM mission (Verhoeve et al. 1992); the only difference is that this CCD has a lower resistivity (20 $\mathrm{ohm} \mathrm{x} \mathrm{cm} \mathrm{instead} \mathrm{of} 1500 \mathrm{ohm} \mathrm{x} \mathrm{cm}$ ). To achieve the optimal $\mathrm{p}^{+}$layer distribution, the energy of the implanted ions was only $10 \mathrm{KeV}$, the concentration was $10^{14}-10^{15} \mathrm{~cm}^{-2}$ and the implanted angle of incidence was increased with respect to the typical angle of $7^{\circ}$. to avoid channeling of implanted boron to great depth: in such a way, the peak of the ion distribution should be, theoretically, at only about $0.05 \mu \mathrm{m}$ inside the CCD. It is important to note that in the last CCD, EEV has applied the "anodic etching" after laser annealing. At a scan time of $100 \mu$ s per pixel all the CCDs show a readout noise smaller than 10 electrons rms.

\section{EXPERIMENTAL SYSTEM}

To evaluate the CCDs performances in the $300-11000 \AA$ region, two different test facilities have been used. One performs QE measurements in the $300-2500 \AA$ range and is located in Padua (Department of Electronics and Informatics) (Bonanno et al. 1992, Naletto et al. 1992) and the other is located in Catania (in our Observatory) and can characterize cooled CCDs in the 1150-11000 $\AA$ range. In these laboratories measurements of the response uniformity are also possible (REFERENCES 2) [11,12]XXXX

The vacuum facility used for the EUV spectral range measurements is shown schematically in Fig. 2: it consists essentially in a normal incidence monochromator and a calibration chamber.

The monochromator has a Johnson-Onaka configuration with an $f / 10$ aperture and a toroidal reflection grating $(600 \mathrm{l} / \mathrm{mm}, \mathrm{Pt}$ coated). The entrance and exit slit are fixed and are $100 \mu \mathrm{m}$ wide and three mm long. It is pumped by a turbomolecular pump which allows it to 


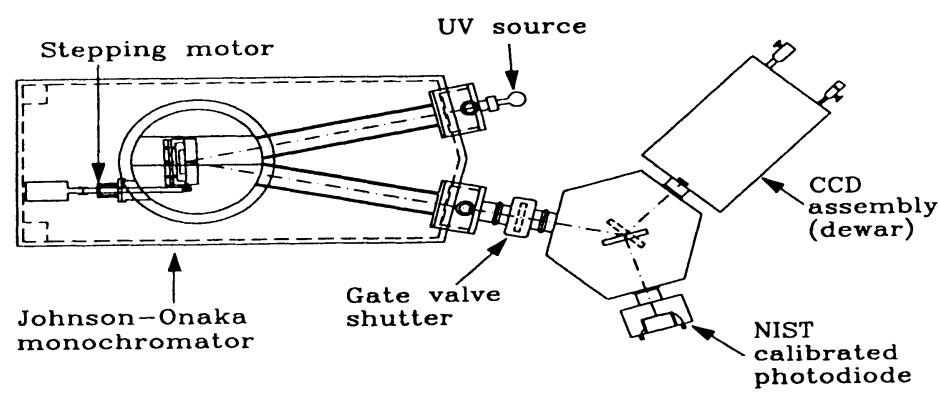

Fig. 2. Scheme of the calibration facility used for the QE evaluation in the EUV.

reach a pressure of about $2 \times 10^{-6}$ mbar. As sources, both a 30 Watt Hamamatsu Deuterium lamp sealed with a MgF2 window emitting in the $1150-2500 \AA$ spectral range and an open hollow cathode lamp to cover the range down to $300 \AA$ have been used. The lines used are 304 $\AA ̊$ (He II), $461 \AA$ (Ne II), $584 \AA$ ( He I), $735 \AA$ (Ne I) and $920 \AA$ (Ar I). The calibration chamber accommodates a rotating $\mathrm{Pt}$ coated toroidal mirror mounted at its center which can focus the monochromatized beam on two symmetric positions, where both the CCD and the calibrated photodiode are mounted. To evaluate the QE, a pair of NIST calibrated photodiodes have been used: one sealed for the 1164-2537 $\AA$ range and the other windowless working in the 50-1216 $\AA$ region.

The system used for the 1150 - $11000 \AA$ spectral range measurements is shown schematically in Fig. 3: it is based essentially in a precision 0.39 -meter focal length vacuum monochromator. This last, thanks to a triple grating turret that can accommodate gratings blazed at appropriate wavelengths, allows good and automated measurements in the above mentioned operating range. Some off-axis paraboloidal mirrors are used to focus the beam on the monochromator. As sources, we use a Tungsten lamp to cover the $4000-11000 \AA$ region and a 150 Watt Deuterium lamp sealed with a MgF2 window emitting in the $1150-3000 \AA$ spectral range. To cover the 3000 - $4000 \AA$ range, we actually use some spectral lamps, but in the future a Xenon lamp will be used. A movable plane mirror selects the source. To evaluate the QE of the CCDs in the spectral range of interest, three NIST calibrated photodiodes have been used: one sealed with a MgF2 window for the $1164-2537 \AA$ range and the others, a quartz window working in the $2000-11000 \AA$ region.

Actually, we use the system assembled on an optical bench without the vacuum chamber reducing the operating wavelength range to $2000-11000 \AA$. The system configuration allows simultaneous measurements to be made on both detector and calibrated photodiode, reducing the errors due to the source variability during the measurements.

The CCD controller used for these measurements has been made in our laboratory (Bonanno and Di Benedetto 1990) and in the future we will use the one designed for the Italian National Telescope "Galileo" (see Bonnano (1995) for the essential characteristics). The acquired images are analyzed using two packages, IDL and IRAF running in Unix-machine workstations. 


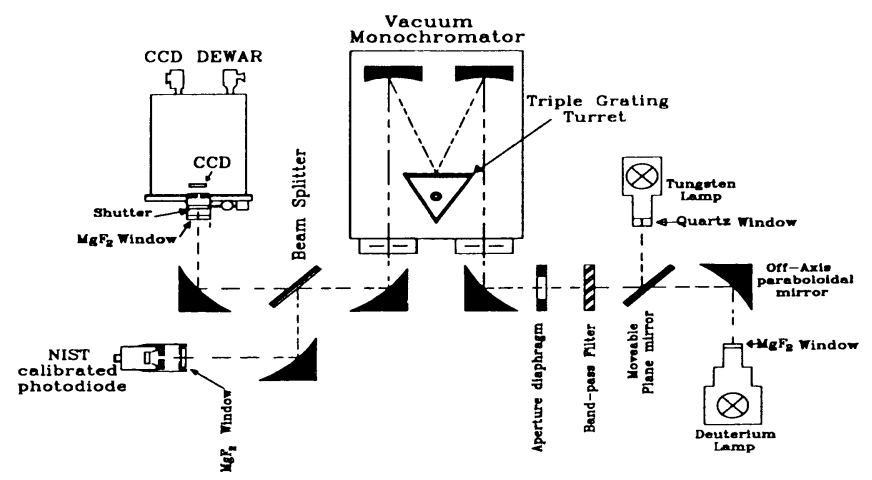

Fig. 3. Scheme of the calibration facility used for the QE evaluation in the $1150-11000 \AA$ spectral range. The system is mounted on an optical bench and is presently used for the 2000 $-11000 \AA$ spectral range because the optical components are not housed in a vacuum chamber.

\section{QUANTUM EFFICIENCY MEASUREMENTS}

The QE measurement is conceptually easy: it is necessary to compare the CCD signal (electrons) with the signal measured by a calibrated photodiode at each wavelength. But the real measurement is not so simple, particularly at wavelengths shorter than $2000 \AA$. Since the sources and their characteristics are different for the regions above and below $1200 \AA$, different methods of evaluating the $\mathrm{QE}$ are adopted for the various cases in the two laboratories (Bonnano et al. 1992, Naletto et al. 1992). Particular care has been taken in the evaluation of the CCD conversion factor $F$, expressed in $e^{-/ D N}$, because the accuracy of this parameter influences all data. It must be pointed out that in the UV spectral region the QE of CCDs is defined as the product of the effective $C C D Q E$; that is, the fraction of incident photons producing one or more $e^{-h}$ pairs that are collected in the CCD pixel wells, multiplied by the so called electron yield; that is, the number of $e^{-h}$ pairs produced per interacting photon (usually it is assumed that the $\mathrm{e}^{-\mathrm{h}}$ pairs are generated by an energy of $3.65 \mathrm{eV}$ ).

\section{$4.1300-2500 \AA ̊$ Spectral Range}

The results of the QE measurements in the $300-2500 \AA$ region are shown in Fig. 4 and refer to data acquired about one hour after the cooling of the CCDs.

The measured QEs of both front-illuminated and back-illuminated coated CCDs, have a similar behavior. They show values greater than $10 \%$ at longer wavelengths and a minimum of the order of $1-1.5 \%$ in the $700-1200 \AA$ range, and both show an increase at the shortest wavelength. A different behavior can be noted on the two ion-implanted devices: first of all, they show a QE greater than $10 \%$ in a larger spectral range $(900-2500 \AA)$, at wavelengths shorter than $700 \AA$ the QE increases quickly and in particular, the "etched" one has a QE greater than $60 \%$ at $304 \AA$. A difference of $25 \%$ can be noted between the two ion-implanted CCDs in the $400-2000 \AA$ that drops to $10 \%$ at longer wavelengths. Moreover, it is very interesting to observe that the two $\mathrm{QE}$ curves converge in the UV region: this fact agrees fairly 


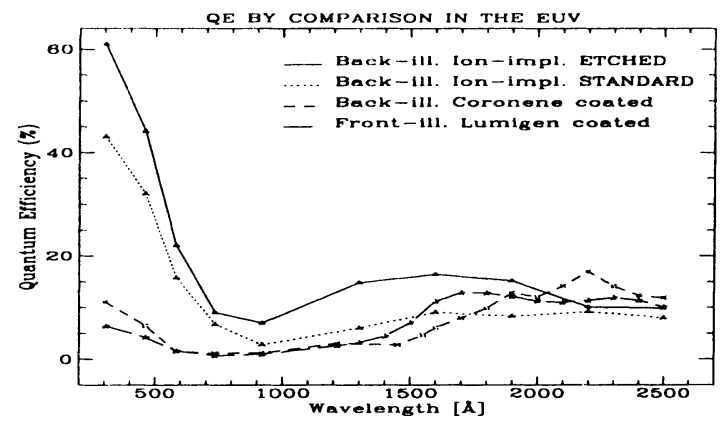

Fig. 4. Comparison between the quantum efficiency data for the various CCDs in the 300 $2500 \AA$ Å spectral range.

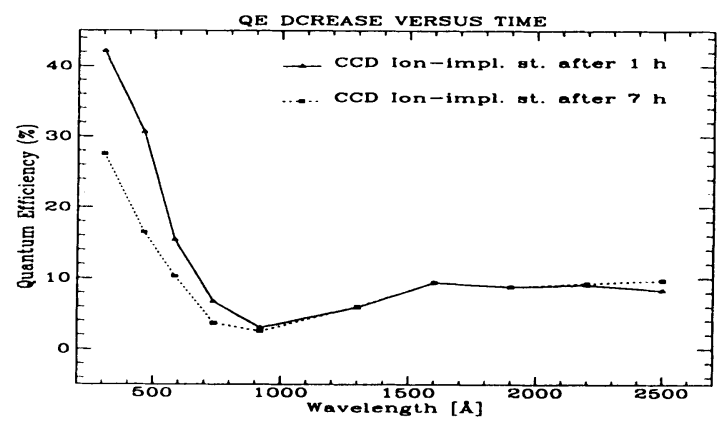

Fig. 5. Quantum efficiency decrease vs time for the thinned back-illuminated ion-implanted "standard" in the $300-2500 \AA$ spectral range.

well with the QE measures obtained in the range 2500 - $11000 \AA$ (see Fig. 6). The minimum values are showed in the range between $700 \AA$ and $1200 \AA$ and are $10 \%$ for the "etched" and $7 \%$ for the "standard".

During the QE measurements, a rather interesting phenomenon has been observed In practice, the $\mathrm{QE}$ decreases in time when the CCDs are cooled, to restart at high values after a cooling cycle. In Fig. 5 the QE data, taken at different times after the cooling of the CCD thinned back-illuminated ion-implanted "standard", is shown. After seven hours the QE at 584 $\AA$ from $15 \%$ drops to $10 \%$.

Some tests were made to find whether this decrease was connected with the UV illumination or it if is due to other effects. There is only one obvious conclusion: the $\mathrm{QE}$ decrease in time is mainly related to the non perfect vacuum conditions. In fact the CCD, when cooled, acts as a cryo-pump and some contaminants present in the vacuum chamber are frozen over the CCD. 


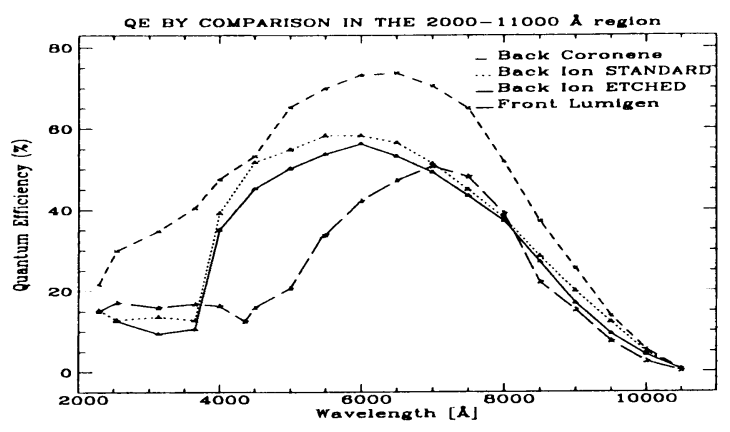

Fig. 6. Comparison between the quantum efficiency data for the various CCDs in the 2000 $11000 \AA$ A spectral range.

\subsection{0-11000 Å Spectral Range}

The results of the QE measurements in the 2000 - $11000 \AA$ region are compared in Fig. 6. For the sake of clarity, we decided not to show the error bars, which are at most not greater than $10 \%$ in the UV region, and much lower at the other wavelengths.

In the 4000 - $10000 \AA$ range all the CCDs, either front- or back-illuminated, show a "typical" QE. In contrast, in the 2000 - $4000 \AA$ range we note a different behavior for the various CCDs. The QE of the front lumigen $\mathrm{CCD}$ is higher than $15 \%$ and is almost constant in that spectral range. The back coronene coated CCD shows a higher QE. But, I have to point out that, unfortunately, the $\mathrm{QE}$ decreases with the number of times the $\mathrm{CCD}$ is operated. The two curves of the back ion CCDs are slightly shifted within a $10 \%$ difference, and, being both within their error bars, we can conclude that they are comparable. Thus, from these results we can say that the "anodic etching" technique improves the QE mainly in the EUV spectral region, leaving it practically unchanged at longer wavelengths.

\section{UNIFORMITY MEASUREMENTS}

To study the uniformity of the CCDs response and its color dependence we used a system constituted essentially by an integrating sphere that is illuminated through interferential filters by different sources in the $2000-11000 \AA$ range. Instead, in the $300-2500 \AA$ range, a ground glass inclined at $45^{\circ}$ to collect the diffuse radiation of the beam coming out from the monochromator is inserted in the calibration chamber. To be sure of the validity of this method, we have been checked that the fluorescent contribution to diffuse light due to the ground glass is negligible with respect to that of the UV.

Here we report the analysis at only two UV wavelengths: $\lambda=1600 \AA$ and $\lambda=2200 \AA$ for the front-illuminated CCD and the back-illuminated ion-implanted "standard" CCD. The pixel to pixel variations have been estimated by dividing the CCD areas into $10 \times 10$ pixel subareas (to minimize the large-scale variations due to the source), and evaluating the mean signal 

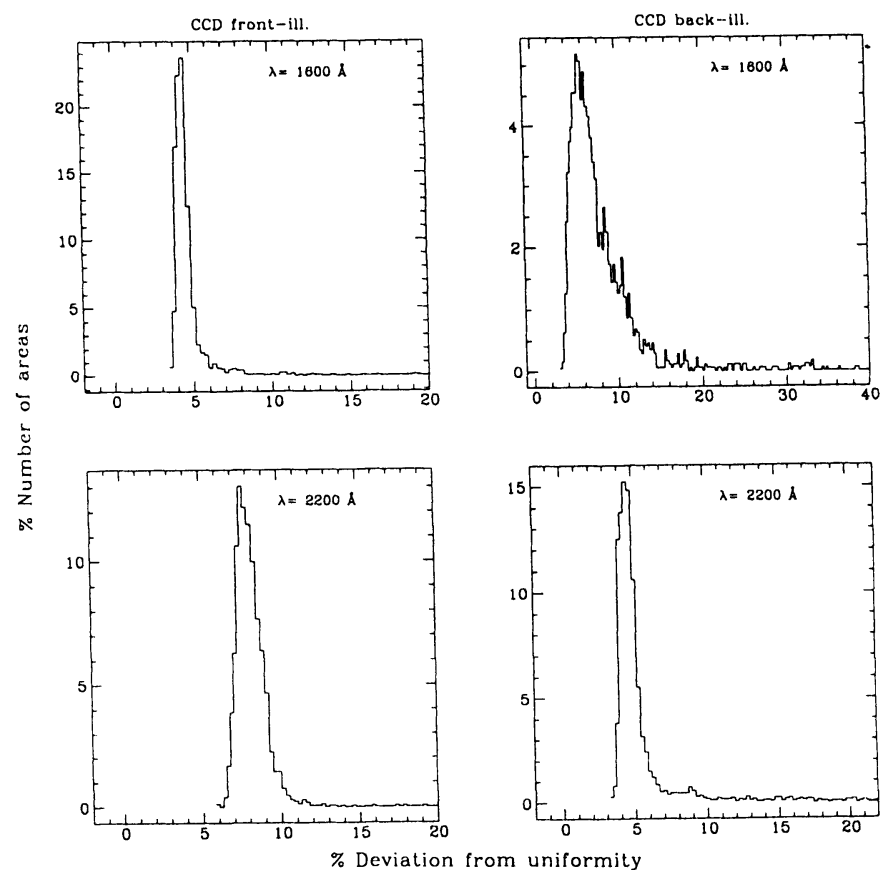

Fig. 7. Deviation of uniformity at $\lambda=1600 \AA$ and $\lambda=2200 \AA$ for the front-illuminated CCD and the back-illuminated ion-implanted "standard" CCD.

$\mathrm{m}$ and the standard deviation $\sigma$ for each sub-area. The deviation from uniformity in any subarea is given by the ratio $2 \sigma / \mathrm{m}$.

Fig. 7 shows the histograms relating the number of the sub-areas to their deviation from uniformity. As can be noted, the front-illuminated CCD shows an opposite behavior with respect to the back-illuminated ion-implanted "standard" CCD. While at $\lambda=1600 \AA$ almost the totality of the considered subareas of the front-illuminated CCD have a narrow distribution around $5 \%$ of non-uniformity, at $\lambda=2200 \AA$ the distribution is enlarged and around $8 \%$ of uniformity. Exactly the contrary is observed for the back-illuminated ion-implanted "standard" CCD. This is mainly due to the absorption depth of the materials at these wavelengths.

\section{CONCLUSIONS}

The QE measurements of the selected CCDs show that both the techniques of coating the CCD surface with some scintillators and of ion-implantation followed by laser annealing are effective in having a rather good QE in the UV spectral region.

The coated CCDs have shown a reasonable QE in the region in which the scintillator efficiently converts the UV radiation, while at shorter wavelengths they show a rather poor $\mathrm{QE}$. Furthermore the QE depends on the number of times the CCD is operated.

The ion-implanted CCD has shown a very high QE, especially at the shortest wavelengths, and a uniformity of response which is good at longer wavelengths; this was an unexpected 
result, so other measurements have to be done to better understand it. The etching process is used to increase the EUV sensitivity of thinned back-illuminated and ion-implanted CCDs. In particular, since QE values as high as $70 \%$ have been measured at the shortest wavelengths, taking into account also the electron yield and the very low noise shown by currently available devices, it is possible to assert that in the spectral range below approximately $600 \AA$ these detectors can work practically in a near photon-counting regime.

The problem of the QE decrease with time, due to residual contaminants inside the vacuum chamber, still remains, but it can be strongly reduced by improving the vacuum and by operating the devices at higher temperatures permitted by new devices that can operate in "inverted mode". In fact Multi Pinned Phase (MPP) devices do not need to be cooled to very low temperatures, thus avoiding the condensation of the contaminants over the sensitive surface. A more accurate evaluation of the QE of MPP CCDs in the EUV will be done in the future.

In conclusion, the results obtained show the real possibility of adopting in the near future these devices as optimal image sensors in the UV and EUV spectral range. In this respect, an effort is already in progress, both as regards the development of new CCD manufacturing technologies by EEV, and the improvement in the test facilities available in our laboratories.

\section{REFERENCES}

Bailey, P., Castelli, C., Cross, M., van Essen, P., Holland, A., Jansen, F., de Korte, P., Lumb, D., Pool. P. and Verhoeve, P. 1990 Proc. SPIE 1344, 356

Bonanno, G., Bruno, P., Consentino, R., Bortoletto, F., D’Alessandro, M., Fantinel, D., Balestra, A. and Marucci, P. 1995 in IAU Symposium No. 167, New Developments in Array Technology and Applications, A. G. D. Philip, K. A. Janes and A. R. Upgren, eds. Kluwer Academic Pub., Dordrecht, p. 319

Bonanno, G. and Di Benedetto, R. 1990 PASP 102, 835

Bonanno, G., Naletto, G., and Tondello, G. 1992 ESA Special Publication, 356, 233

Brinkman, A. C., Aarts, H. J. M., Burt, D. and Pool, P. 1992 ESA Special Pub. 356, 75

Janesick, J. R., Campbell, D., Elliot, T. and Daud, T. 1987 Opt. Eng. 26 (9), 852

Bredthauer, R. A., Chandler, C. E., Janesick, J. R., McCurnin, T. W. and Sims, G. R. 1988 in Instrumentation for Ground-Based Optical Astronomy, Present and Future, L. Robinson ed., Springer-Verlag, p. 486

Janesick, J. R., Elliot, T., Daud, T., McCarthy, J. and Blouke, M. 1985 in Solid States Imaging Arrays, K. N. Prettyjohns, and E. L. Dereniak, E. L. eds., Proc. SPIE, 570, 46

Janesick, J. R., Elliot, T., Dingizian, A., Bredthauer, R. A., Chandler, C. E., Westphal, J. A. and Gunn, J. E. 1990 Proc. SPIE 1242, 223

Janesick, J., Elliot, T., Fraschetti, G., Collins, S., Blouke, M. and Corrie, B. 1989 Proc. SPIE 1071, 153

McCarthy, K. J. and Wells, A. A. 1992 Proc. SPIE 1743, 211

Naletto, G., Tondello, G., Villoresi, P., Bonanno, G., Cali, A., Di Benedetto, R. and Scuderi, S. 1992 Proc. SPIE, 1743, 199

Stern, R. A., Catura, R. C., Blouke, M. N. and Winzenread, M. 1986 Proc. SPIE 627, 583

Verhoeve, P. W. A. M., den Boggende, A. J. F., Jansen, F. A., de Korte, P. A. J., Burt, D., Castelli, C. and Holland, A. D. 1991 in Astronomical Application, Conf. on Photoelectronic Image Devices 1991 - Section 1, p. 25 
Verhoeve, P. W. A. M., Jansen, F. A., de Korte, P. A. J., den Boggende, A. J. F., Bonanno, G., Cali, A., Di Benedetto, R. and Scuderi, S. 1992 Proc. SPIE 1743, 223

\section{DISCUSSION}

STOVER: At Lick we are investigating two additional techniques to address the problems of backside treatments. First, we are having wafers fabricated which have the highly doped boron layer built into the silicon. This makes thinning the CCD easier. In addition, it should provide backside accumulation properties similar to ion implanting with annealing. Second, we are planning to experiment with n-type CCDs. CCDs made with this type of material will not have the backside accumulation problems of standard CCDs.

BONANNO: Very well. I agree completely to experiment with n-type CCDs. I hope we will see the results of these processes in the future.

COHEN: Since no one from JPL appears to be here, I should like to point out that Paula Grunthaner of the Microdevices Lab is working on this problem and is using a different technique. This has some advantages and some disadvantages - the process requires very high vacuum and cleanliness and is inconsistent with most commercial production. But the $\mathrm{QE}$ waves are spectacular. Paula expects to distribute a few CCDs treated in this way for testing soon.

BONANNO: OK. I will read in the future the results of the $\mathrm{QE}$ measurements.

JORDEN: On the graph showing QE of the four CCDs over the 2000-10000 $\AA$ range - why does the coronene coated thin device show such a high peak $(600 \mathrm{~nm})$ response of $80 \%$ (relative to $60 \%$ on other thin ones)?

BONANNO: I think that the coating acts as an antireflection coating at these wavelengths.

CULLUM: I am not clear on your reference to ESO-type coronene coatings. ESO has not used coronene coatings, only laser dye coatings on an acrylic base. Could you clarify what these coatings consist of?

BONANNO: EEV said that the coating applied on the thinned back illuminated CCD is coronene and the method utilized is similar to the one used from ESO. This is an older technique that is very different from the phosphor sublimation under vacuum conditions. 